\title{
¿POR QUÉ SE ESTUDIA TRADUCCIÓN E INTERPRETACIÓN EN ESPAÑA? EXPECTATIVAS Y RETOS DE LOS FUTUROS ESTUDIANTES DE TRADUCCIÓN E INTERPRETACIÓN Eugenia Arrés López. Traductora y moderadora de ForoTraducción Elisa Calvo Encinas Grupo de investigación Avanti, UGR
}

\section{ABSTRACT}

This article aims to present certain conclusions on a very specific group, as is the profile of students wishing to study Translation and Interpretation. Therefore, the aim is to make an initial outline of the expectations and motivations of future Translation and Interpretation candidate students, by trying to identify their needs, fears, false conceptions and expectations.

KEYWORDS: student, translation, interpretation, expectations, needs.

\section{RESUMEN}

El presente artículo pretende exponer algunas conclusiones, centradas en este caso en un colectivo muy específico, como es el perfil del estudiante que desea estudiar TeI. Se pretende aquí, por tanto, realizar un boceto inicial de las expectativas y motivos de los estudiantes candidatos a futuros licenciados de TeI, intentando identificar sus necesidades, sus miedos, sus falsas concepciones y sus expectativas.

PALABRAS CLAVE: estudiante, traducción, interpretación, expectativas, necesidades.

\section{INTRODUCCIÓN}

Aunque Bolonia propone al estudiantado como clave del proceso educativo, no deja de sorprender que los intentos de acercamiento institucional a la realidad del estudiantado en general y a la realidad del estudiantado de Traducción e Interpretación (en adelante, TeI), en particular, son escasos. Así, en pleno proceso de reformas curriculares, poco sabemos de aspectos tan 
importantes como cuáles son los motivos que llevan a los estudiantes a elegir esta carrera y las expectativas que los estudiantes tienen sobre los estudios de TeI o sobre su proyección profesional. Si bien motivaciones y expectativas son parámetros de protagonismo escaso en el entorno tradicional de la universidad española en general, las conexiones entre dichos conceptos y el éxito del proceso de aprendizaje cada vez son más destacados en las corrientes contemporáneas de Educación Superior (por ejemplo, Biggs, 2003: 57-64 o Fry et al. 2003: 62-75; en un contexto de traducción: Kiraly, 2000; en un contexto de TeI en España, Kelly, 2005: 49). Con la información que tenemos hasta ahora, algunos docentes y observadores intuyen los problemas que puede generar un choque entre expectativas estudiantiles y planteamientos curriculares o didácticos.

Por otra parte, Internet se revela como un testigo de excepción de las necesidades reales del estudiantado y como la principal herramienta de búsqueda de información actual, al menos en frecuencia e intensidad de uso de recursos como foros, blogs, páginas personales, etc.

De estos dos enfoques, a saber, análisis de la situación de los estudios de TeI en España desde la perspectiva del estudiantado y comunidades virtuales dirigidas al colectivo de la traducción e interpretación, surge el presente trabajo. A lo largo de los años 2005 y 2006, las autoras coinciden en el tiempo en dos proyectos con diferentes propósitos pero con conclusiones coincidentes. Por un lado, Eugenia Arrés pone en marcha Forotraducción, como iniciativa para la creación de un punto de encuentro entre individuos interesados en los estudios de TeI, estudiantes de la Licenciatura y licenciados, con el fin de constituir una comunidad de intercambio desinteresado de información académica y profesional. Para extraer la información, se ha realizado un análisis cualitativo de las respuestas, conforme a unos criterios simples de categorización basados en la selección de las dudas relevantes planteadas por foreros cuyo perfil se adapta al relevante para el estudio (estudiantes que se plantean el acceso a la carrera). La información que se recopila se ha extraído de los más de 2000 mensajes enviados por los 330 miembros de la comunidad.

Por otro lado, Elisa Calvo realiza su tesis doctoral basada en un análisis curricular de los estudios de TeI desde la perspectiva del propio estudiantado, dentro del grupo AVANTI, bajo la dirección de la profesora Kelly. La metodología aplicada es la propia de la investigación exploratoria no experimental (Grotjahn, 1987) con cuestionarios dirigidos a alumnos de primer curso, recién llegados (primer trimestre), procedentes de seis facultades extraídas de la población total, a saber, UGR, USAL, UPV-UEH, UPCO, UAX y UJI. Se obtuvieron un total de 326 cuestionarios, que desde el punto de vista del tamaño de la muestra supone un porcentaje satisfactorio (en torno a un $7 \%$ de una población total de $+/-2100$ estudiantes). 
La presente comunicación pretende exponer algunas conclusiones, centradas en este caso en un colectivo muy específico, como es el perfil del estudiante que desea estudiar TeI. Se pretende aquí, por tanto, realizar un boceto inicial de las expectativas y motivos de los estudiantes candidatos a futuros licenciados de TeI, intentando identificar sus necesidades, sus miedos, sus falsas concepciones y sus expectativas.

\section{CONTEXTO}

En España se licencian al año unos 1400 estudiantes de TeI y la cifra va en progresivo aumento. El número de plazas ofertadas en primer curso ha experimentado un incremento sustancial, especialmente si se tiene en cuenta que en los últimos diez años se ha pasado de contar con menos de diez centros de TeI, entre públicos y privados, a tener un total de 25 centros de TeI repartidos por el país. TeI ya no es una carrera minoritaria. Frente a su demanda en incremento, se detecta una tendencia opuesta en la mayoría de las demás carreras, especialmente las consideradas tradicionalmente de "letras". Así, la carrera parece estar, en cierto modo, de moda (Kelly, 2005:50) y gozar de una mejor reputación entre los alumnos que otras carreras relacionadas con los idiomas, por diversas razones que intentaremos analizar. Kelly (2005: 66) contempla la posibilidad de que TeI esté aglutinando a todos aquellos estudiantes que, ante la falta de otras carreras alternativas a la filología en las que los idiomas se enfoquen desde puntos de vista aplicados al mercado y a la sociedad (Lenguas Aplicadas, Comercio Internacional), opten por TeI como única opción posible. En esta misma línea, Mayoral (2005) apunta:

La inadecuación y crisis de las filologias, el prestigio de la actividad de la traducción, la inexistencia en nuestro país de estudios de lenguas aplicadas, etc. ha ocasionado que la demanda de estudios de traducción haya desbordado incluso la gran demanda existente del mercado profesional e incluya (...) a cualquier estudiante que pretenda dedicarse profesionalmente a actividades relacionadas con las lenguas extranjeras y no solo a los que quieran dedicarse a la traducción e interpretación. (Mayoral Asensio: 2004, en prensa)

Partiendo de estas observaciones de la realidad, pasamos a cotejar los datos de los que disponemos y observamos que tienen un claro reflejo en las dos fuentes que aquí se presentan. Hemos recogido de forma sintética una lista de las apreciaciones que se valorarán, de forma más específica:

1. Muchos estudiantes eligen TeI porque quieren estudiar idiomas de una forma aplicada, pero no todos tienen claro que quieren ser traductores e intérpretes (Kelly, 2005: 50, 65). En muchos casos, se rechaza la filología por tener un contenido que consideran poco práctico y salidas laborales que se asocian prácticamente de forma exclusiva a la docencia, una actividad que parece no atraer a buen número de estudiantes. 
2. Aquellos que sí quieren ser traductores e intérpretes con certeza, están confusos en torno a los perfiles profesionales del traductor e intérprete, dándose demasiado peso, con respecto a su incidencia como nicho de empleo real, a los perfiles más atractivos a priori (Kelly, 2005: 50).

3. Muchos estudiantes se ven movidos por un interés en viajar (Kelly, 2005: 50, Calvo, en prensa) y por el estilo de vida que asocian a los licenciados de esta carrera (movilidad, cosmopolitismo).

4. Muchos estudiantes no se esperan los contenidos de la carrera en general y los enfoques curriculares de algunas facultades en particular.

5. Muchos estudiantes temen que las salidas profesionales no se ajusten a lo que ellos esperan. Esto tiene traducción en varias direcciones. Por un lado, están sus expectativas de encontrar trabajos de alto perfil económico y/o social. Por otro lado, está su deseo de "no ser profesor de idiomas" (Kelly, 2005: 50). Una tercera tendencia está representada por los que tienen claro que quieren ser traductores e intérpretes y temen no poder llegar a conseguirlo por las condiciones del mercado.

6. Muchos estudiantes temen los restringidos sistemas de acceso y el grado de exigencia de la carrera, especialmente en lo relativo a los idiomas.

\section{RESULTADOS: MOTIVACIONES, EXPECTATIVAS}

\subsection{MOTIVACIONES}

- Idiomas, pero de una forma aplicada (Kelly, 2005: 50, 65; Mayoral, 2004)

A) Impresiones extraídas de Forotraducción (noviembre 2006)

"Me encantaría estudiar Traducción; antes me planteé Filología Inglesa pero no me llena". "Yo no quería hacer Filología porque no me gustan nada las salidas que tiene".

(Extraído de Forotraducción, 27.11.2006)

Una de las conclusiones que se extraen entre los comentarios vertidos en el foro, es que a la hora de elegir los estudios, muchos alumnos no sienten un impulso vocacional por un área de conocimiento en concreto. Ante esto, muchos eligen estudios que respondan a un área de estudio que conocen de la Secundaria y que se les da bien y/o les gusta. Así, comienzan por considerar la idea de estudiar, preferiblemente en la universidad, "algo relacionado con los idiomas". Entre las titulaciones que más les interesan se encuentran la diplomatura en Turismo y, sobre todo, las distintas filologías, destacando en incidencia la filología inglesa. No obstante, por lo general, los miembros del foro consideran que la licenciatura en TeI ofrece ciertas ventajas sobre el resto 
de estudios que barajan que, sumadas, harán que se decanten por nuestros estudios:

1) Se detecta un cierto rechazo a las salidas que les ofrecen otras carreras relacionadas con los idiomas. El ejemplo más claro es la docencia, en el caso de las filologías y, también, el trabajo en un hotel o una agencia, en el caso de la diplomatura en Turismo. Creen que la formación en general y, más concretamente, en lenguas extranjeras, que se recibe en TeI es más amplia y especializada. Se han vertido algunas valoraciones comparativas en las que Turismo, por ejemplo, se rechaza por tener menos horas de idiomas y una enseñanza demasiado restringida al sector turístico. Frente a la filología, los principales argumentos son que TeI se aleja de los estudios lingüísticos tradicionales con los que ellos asocian a la filología, más abstractos o teóricos que aplicados. Asimismo, la posibilidad de trabajar con más de una lengua, frente a la filología con una sola lengua central, también es para ellos una ventaja. Además, asocian la formación más práctica y especializada con más y mejores salidas profesionales.

2) La Licenciatura en TeI parece estar "de moda". Para los estudiantes, supone una alternativa a las filologías, cuyas perspectivas laborales serán de mayor categoría (puestos de mayor responsabilidad y más atractivos que "ser profesor toda la vida”), que huye de lo convencional (un perfil de profesionales que viajan y tienen una gran "cultura general", conocedores de muchos campos temáticos). Muchos de los estudiantes que tienen una nota media alta en Secundaria y no presentan una vocación clara se sienten atraídos por los estudios de TeI, ya que presuponen que esta nota alta se traducirá en un sueldo alto y buenas opciones de empleo. Cabe recordar en este punto, que las notas para otras carreras con contenido de idiomas son bastante más bajas que en TeI (por ejemplo, Turismo). La diferencia es mucho más extrema en las filologías, donde en muchas ocasiones no cubren su cupo de plazas (lo que se refleja en una nota de corte de 5 en muchos centros). Las notas altas se interpretan entonces como estudios de élite. Así, se muestran altamente decepcionados o sorprendidos cuando se incluyen posts que hacen referencia a la dureza del mercado laboral en algunos aspectos. También se registran comentarios en los que se establecen paralelismos o se refleja interés con respecto a otras titulaciones universitarias que gozan de una buena valoración según diferentes criterios. Por lo tanto, asocian la nota de corte a la dificultad y al prestigio (Medicina, algunas Ingenierías) y, por extensión, a mejores perspectivas profesionales. También parece establecerse una comparación con respecto a otras carreras "de moda" (Comunicación Audiovisual), con una gran demanda académica, que adquieren prestigio, entre otras cosas, por el efecto "revalorizador" del sistema de numerus clausus. Tras este análisis, cabe destacar que, en una alta proporción, los alumnos que se enfrentan al escollo de no poder acceder a TeI, sea por falta de nota media, sea por no pasar la prueba de 
acceso, acaban "reciclados" en filología o turismo. De entre ellos, muchos lo hacen pensando en intentar acceder a la licenciatura en TeI posteriormente.

B) Resultados del cuestionario

Cabe recordar que las muestras son aquí diferentes: los comentarios del foro se han extraído en su mayoría de futuros posibles alumnos de TeI y alumnos recién entrados, mientras que la tesis, tal y como ya se ha explicado, se refiere a alumnos que ya están en primer curso (más concretamente, en el primer trimestre del primer curso (2005). Para empezar, en los resultados del cuestionario se muestra que un 93,9\% de los estudiantes sondeados afirman que TI era su carrera preferida a la hora de entrar a la universidad. Este dato está relacionado con el hecho de que casi todos los estudiantes acceden a la carrera recién salidos de la Educación Secundaria y con calificaciones que tienden a ser altas. Por este motivo, no suelen tener problemas de acceso a su primera elección en las facultades con numerus clausus. Esta tendencia también encaja con el perfil de rendimiento de la mayoría de los que superan un examen de acceso. En carreras con sistemas de acceso menos restrictivos, este tipo de indicador se invierte, con muchos más estudiantes que hubieran preferido estudiar otra carrera. En cuanto a la pregunta dedicada a averiguar qué otras carreras despertaban su interés al acceder a la universidad, en total, un 60,4\% de alumnos optó en su elección universitaria únicamente por carreras en las que los idiomas juegan un papel esencial. Este dato se desglosa en un 23,01\% de la muestra para el que la única opción posible de acceso a la universidad fue TeI, un 32,21\% cuya opción alternativa a la TI era la filología y un 5,2\% que optó por TI y turismo. En cuanto a por qué se decidieron por TeI, se barajan distintos motivos:

1. "Porque me gustan los idiomas" mueve a un $98,8 \%$ de los estudiantes. Asimismo, un $94,8 \%$ se siente motivado por la razón "porque quiero aprender idiomas en la carrera".

2. "Porque quería hacer una carrera con idiomas, ya fuese filología o Tel" arroja la conclusión de que la mayoría de los estudiantes sondeados querían hacer una carrera relacionada con los idiomas en cualquier caso (65\%), ya fuera Tel o filología, con preferencia por Tel. "Porque quería hacer una carrera con idiomas, pero no Filología" fue motivo de elección para un significativo $35 \%$ del total de los alumnos.

3. Por otro lado, se muestran menos interesados por el contenido curricular de la carrera

Varias conclusiones pueden obtenerse de este resumen de datos. Como es obvio, la mayor parte de los estudiantes accede por su interés por los idiomas, aunque la gran mayoría de ellos desea aprenderlos durante la carrera. Cabe considerar si el planteamiento curricular de la carrera que parte de la base de que los estudiantes ya tienen un nivel suficiente de su lengua B, es realista o no. Por otra parte, la tendencia a escoger TeI porque no les interesa Filología, sí es significativa, aunque no mayoritaria, con más de un tercio de los alumnos 
que identifica este motivo. Asimismo, esta tendencia queda confirmada, si no mayoritariamente, sí de forma significativa, cuando se les pregunta cuál es el trabajo que menos desearían tener que realizar. Así, la docencia es el trabajo más rechazados a priori, con un 25,5\% de estudiantes que especifica de forma explícita que no desea trabajar en este sector. Finalmente, casi un tercio confiesa haber elegido la carrera porque tenía una nota lo bastante alta para entrar.

- Idiomas como puente a un cierto estilo de vida o profesión

A) Impresiones extraídas de Forotraducción (noviembre 2006)

"Eso de estar todo el día con los idiomas me encanta. Además, supongo que también habrá posibilidades de viajar". "Y luego cuando se acaba la carrera, ¿qué tal?... Habrá posibilidades de hacer unas oposiciones para la ONU".

"A mí, lo que más me gustaría es trabajar en traducción literaria aunque lo de doblaje y subtitulado también". (Extraído de Forotraducción, 27.11.2006)

Entre los miembros del foro, pocos son los que conocen a priori los estudios de TeI o la profesión de traductor o intérprete antes de decidirse completamente por la licenciatura en TeI. Una vez descartada la docencia, no tienen una idea clara de cómo se pueden aplicar los idiomas en otros campos, ni de qué asignaturas encontrarán en los estudios de TeI. Casi toda la información que reciben se encuentra en Internet o se la ha facilitado gente de su entorno que en realidad no tiene contacto directo con la traducción. La ausencia de una perspectiva de primera mano de la licenciatura o del trabajo realizado por traductores o intérpretes hace que creen un referente profesional algo confuso pero que responde a un patrón que suele repetirse en el foro:

1) Intérprete: conocen su figura en mayor medida que la del traductor gracias a la información que reciben en los medios de comunicación. La aparición de la figura del intérprete en cine y televisión, por ejemplo, asocia su labor profesional a trabajos de perfil alto (en importantes instituciones y para clientes destacados): los intérpretes realizan su trabajo en ruedas de prensa con políticos, deportistas, actores, escritores, etc., o en organizaciones internacionales como la ONU, la UE, etc.

2) Traductor: consideran, casi en exclusiva, el perfil literario o audiovisual (por afinidad con sus intereses o porque se trata de un perfil con mayor reconocimiento social). Pocos conocen que muchos traductores realizan su trabajo frente a un ordenador durante largas jornadas, ni los distintos perfiles y tipos de textos especializados a los que se puede aspirar.

Especialmente debido a la imagen que tienen de los intérpretes y por asociación con el aprendizaje de idiomas, presuponen que los intérpretes (y, por extensión, los traductores) trabajan en el extranjero y viajan mucho. Se registran numerosos posts sobre cómo funcionan los intercambios y también 
sobre opciones para viajar al extranjero durante los veranos para trabajar o estudiar, incluso antes de acceder a la carrera. Con respecto a la movilidad que se ofrecerá en la carrera, su principal preocupación es la financiación. Algunos estudiantes también consultan la conveniencia de estudiar la carrera en el extranjero íntegramente.

B) Resultados del cuestionario

La estructura en ítems del cuestionario ofrece una información más específica en ciertas direcciones pero menos profunda y detallada que la recogida cualitativamente en el foro. Con respecto a lo que se percibe en el foro, el cuestionario incluye un ítem que contempla el interés por viajar como motivación para entrar en TeI. Un 84,2\% considera que este motivo influyó en su decisión de estudiar traducción. En cuanto al grado en que se sienten atraídos por un modo de vida cosmopolita o multicultural, un 95,4\% de los alumnos elige la carrera bajo el motivo "porque me gusta conocer gente de otros países". Asimismo, un 55,8\% de los alumnos está convencido de que los licenciados en TeI podrán aspirar a una buena remuneración y un 86,2\% cree que la carrera tiene buenas salidas profesionales. ${ }^{1}$ Se confirma el interés inicial por la interpretación con un porcentaje de alumnos ligeramente superior que preferiría ser intérprete a traductor en el momento de acceder a los estudios.

\subsection{EXPECTATIVAS}

- Contenido de la carrera

A) Impresiones extraídas de Forotraducción (noviembre 2006)

"Me gustaría saber si en la carrera habrá algo de literatura o de historia, que me apasionan...".

(Extraído de Forotraducción, 27.11.2006)

Las expectativas de los foreros se centran fundamentalmente en lo que esperan encontrar durante sus estudios universitarios y en qué habilidades desarrollarán que puedan servirles en su vida personal y profesional en el futuro. Desean profundizar en los idiomas que ya conocen y descubrir otros nuevos, por lo que la licenciatura en TeI parece brindarles esta oportunidad. También se registran posts en los que destaca el interés por aprender las denominadas "lenguas exóticas", que no suelen ofrecerse, de forma aplicada o

\footnotetext{
${ }^{1}$ Este último aspecto es interesante. Según Calvo (2006), en un análisis de los distintos estudios de inserción existentes, se puede considerar cierto que las expectativas de inserción laboral de los estudiantes son muy positivas. No obstante, al principio de la carrera los estudiantes consideran las salidas profesionales de forma muy positiva y al final de la carrera esta percepción se torna muy pesimista. ¿Qué les hace cambiar de idea, equívocamente, a lo largo de los estudios? Todo a punta a sistemas de orientación profesional claramente deficientes en las diferentes facultades y a la influencia de comentarios sesgados y negativos por parte de algunos profesores y compañeros.
} 
con un nivel alto, fuera de esta carrera. Desean convertir lo que antes era una afición (ver películas o leer libros en inglés, descargar manga japonés subtitulado) o un complemento a su formación (ir a una academia de idiomas) en la base de sus estudios y de su profesión. Dado que las asignaturas de esta titulación se relacionan en su mayoría, de forma directa o indirecta, con el estudio de las lenguas, muchos deducen que al finalizar sus estudios universitarios dominarán a la perfección su lengua $\mathrm{B}$, de forma oral y escrita y esperan llegar a ser "bilingües". Como ya se apunta en el punto anterior, es probable que la forma en la que se enfoca el aprendizaje de idiomas en la carrera, dando por hecho que los alumnos ya vienen con un nivel determinado, resulte en la frustración de esta expectativa. Algunos de ellos, incluso creen que el nivel de las lenguas $\mathrm{C}$ o D se igualará al de la lengua $\mathrm{B}$ al final de la carrera, alcanzando la misma competencia lingüística. Además de los idiomas, gran parte de los alumnos se interesan por otras disciplinas cuyos contenidos esperan encontrar en las asignaturas de TeI, tales como historia o literatura, normalmente relacionadas con la "cultura general" que desean adquirir. Para algunos, esta idea supone un aliciente para el estudio de esta titulación. Para otros, resulta un punto común con las filologías y se muestran contrarios a la idea de una posible inclusión en la carrera, aunque tampoco esperan a priori que los itinerarios de especialización tengan tanto que ver con campos como el derecho, la ciencia o la tecnología. Así, pocos miembros conocen las distintas especialidades dentro de la traducción o la interpretación. Cabe señalar que las facultades de TeI se organizan de dos formas fundamentales: a) multidisciplinarmente, aprovechando asignaturas de campos como el Derecho, la Economía y la Ciencia o Tecnología para cubrir la optatividad y libre configuración (ver Mayoral, online) ${ }^{2}$ y b) monodisciplinarmente, basando los contenidos en asignaturas de filología u otras áreas, que tienen algún tipo de conexión institucional local con TeI, como Humanidades o Documentación. Los foreros parecen encontrar menos chocantes las relacionadas con filología, y a priori no esperan contenidos de áreas que probablemente sean más útiles en su formación, pero no parecen tan directamente relacionadas con lo que ellos esperan, como son Derecho o Ciencia.

B) Resultados del cuestionario

En este caso, el cuestionario registra las principales carencias de orientación, comparables a las conclusiones que se extraen del foro. En la

\footnotetext{
2 "Las universidades intentan contener gastos evitando la multiplicación de las mismas disciplinas o disciplinas semejantes en diferentes centros.(...) Se obliga a que alumnos de diferentes centros asistan a las mismas clases cuando sus asignaturas tienen denominaciones y/o contenidos iguales o semejantes. (...) Aparte de los ahorros económicos (...), las desventajas son evidentes. Difícilmente se encuentran en las respectivas carreras materias tan generales (...) o de nivel tan accesible como las que necesitarían la mayoría de nuestros estudiantes". Mayoral en colección de artículos on-line "Aproximaciones a la traducción", del Instituto Cervantes Virtual, ver referencia completa.
} 
mayoría de los casos muestreados, la información recibida en educación secundaria se refiere a las notas de corte que se requieren y las facultades que ofertan esta carrera, aunque este dato "mayoritario" apenas supera la mitad de los encuestados $(54 \%)$, por lo que la otra mitad ni siquiera recibió información sobre sistemas de acceso, facultades y notas de corte. En cuanto al contenido de la carrera, solo un $23 \%$ fue informado sobre el contenido curricular de la carrera (asignaturas y plan de estudios). Un 31,9\% recibió algún tipo de información relacionada con las salidas profesionales disponibles, aunque a la hora de especificar la utilidad y alcance de dicha información, no queda claro si esa información fue realmente suficiente o fiable. Así, por ejemplo, solo un $8,9 \%$ recibió información sobre lo que gana un licenciado en TI (y no hay constancia de la calidad de la información recibida).

Además de la orientación recibida en Secundaria, los estudiantes se informaron por otros medios. La fuente principal de información es Internet. Un $74,5 \%$ del total de estudiantes muestreados obtuvieron información por esta vía. La siguiente fuente de información es el consejo de familiares o amigos que no están relacionados con el mundo de la traducción e interpretación (48,8\%). En tercer lugar, se recurre a los estudiantes de traducción e interpretación (47,5\%), seguido de los libros sobre estudios y carreras $(39,6 \%)$. Una incidencia menor tienen las consultas en las propias facultades $(27,6 \%)$, jornadas de puertas abiertas de las universidades (19,9\%), la información al respecto que emiten los medios de comunicación y el consejo de traductores e intérpretes profesionales (18,4\%). Lo que más choca en este sentido, es el poco peso que la Universidad tiene a la hora de captar e informar convenientemente a los alumnos. En el cuestionario también se pregunta hasta qué punto se vieron motivados a elegir TeI por la razón "porque me gustan las asignaturas que hay en la carrera". Aquí, un 21,8\% dice que no se siente nada atraído por los contenidos de la carrera y solo un 14\% dice sentirse "muy" atraído por los contenidos propuestos. Este indicador podría estar relacionado con la idea de que una proporción significativa de estudiantes escogen TeI ante la ausencia de otras posibilidades de estudiar lenguas aplicadas, pero sin estar convencidos de querer ser traductores e intérpretes. También puede relacionarse con el hecho de que los contenidos no encajan con el perfil profesional que ellos tienen en mente (relacionado con literatura, especialmente).

\section{CONCLUSIONES:}

Tanto motivaciones como expectativas deberían orientar el diseño de la formación en todos los niveles: asignatura, curso, currículum, estrategias de 
orientación y tutoría, programas de movilidad $^{3}$, etc. La forma en la que afrontan la carrera los estudiantes influye directamente en cómo se sienten de identificados y de cómodos con sus estudios, en las estrategias y actitudes que aplican a la hora de enfrentarse a las situaciones difíciles (por ejemplo, falta de nivel lingüístico, frustración de las expectativas creadas a priori, búsqueda de trabajo al acabar, etc.) y en el índice de abandono de los estudios antes de graduarse. La detección de necesidades formativas del estudiantado, mediante el análisis o la evaluación diagnóstica, ayuda a que el plano académico encaje más positivamente con la experiencia formativa del alumnado. Un alumnado bien informado sobre los estudios que realiza, con conciencia de la finalidad de sus estudios, podrá determinar con mayor precisión los posibles itinerarios dentro de la carrera y tomar decisiones informadas sobre por qué escoger una asignatura y no otra, una especialización u otra. La situación polifacética y compleja que esboza este trabajo, viene representada por las siguientes cuestiones principales, que resumimos a continuación a modo de conclusión:

1. Necesidad de más y mejor orientación. La demanda de información por parte de los estudiantes que desean acceder a TeI tiene su origen en un sistema de información y orientación no todo lo eficaz que cabría esperar en la educación secundaria y en la información orientativa que ofrecen las Universidades y Facultades, no siempre todo lo útil y realista que cabría esperar. Así, encontramos que en los descriptores de salidas laborales de las páginas Web de las distintas universidades, abundan las referencias a los perfiles profesionales "gancho" (traductor literario, en Organizaciones Internacionales, en doblaje, etc.) (Arrés, 2005) y no ajustadas a la realidad de inserción profesional que se registra en los diferentes seguimientos de empleabilidad realizados por varias universidades (Calvo, 2006: 633-649). Sería necesario que las Facultades realizasen un esfuerzo en cuanto a la información que se ofrece a los estudiantes, especialmente en lo relativo a las salidas profesionales (Calvo, 2006: 633-649). Asimismo, la orientación curricular y profesional ya dentro de los estudios, debería traducirse en acciones orientativas desde primer curso, como objetivo curricular general y transversal.

2. Necesidad de adaptar los estudios al perfil real de los licenciados. Esto tendría que ver, especialmente, con el aprendizaje de idiomas. Se haría necesaria una revisión de la adecuación del plan de estudios al nivel lingüístico de la mayor parte de los estudiantes en su lengua B. Cuando la carrera aún era minoritaria y la selección solo por examen en las dos o tres facultades pioneras, es cierto que había un mayor número de estudiantes cuasi-bilingües que además en ocasiones pertenecían a una generación de hijos de inmigrantes retornados a

\footnotetext{
3 Para expectativas y motivación en programas de movilidad, consúltese el proyecto europeo TeMCU en www.temcu.es.
} 
España, cuyo nivel de la lengua extranjera solía ser superior. Pero ahora, quizás el nivel de Secundaria no se presta al currículum actual.

3. Necesidad de una diversificación en las carreras que ofrecen lenguas como elemento principal de formación y aplicación profesional. La carrera de TeI está supliendo el papel que otras titulaciones no existentes deberían realizar (Kelly, 2005: 50). La creación de titulaciones alternativas, o, tal y como se propone desde algunos sectores involucrados en la reforma curricular dentro del proceso europeo, de una titulación genérica en Lenguas Aplicadas con especializaciones en Máster en campos como la Traducción o la Interpretación, tendría como efectos positivos:

a. Una respuesta más clara a las expectativas del estudiantado que en realidad quiere estudiar lenguas pero no necesariamente traducción e interpretación, o no en el grado de especialización actual.

b. Una mayor probabilidad de éxito profesional para aquellos que realmente quieren ser traductores e intérpretes, al enfrentarse a un mercado sin la saturación de graduados de hoy.

c. Un futuro claro para las lenguas en la universidad, con enfoques modernos que dejen atrás las principales causas de la crisis de captación de estudiantes de algunas filologías.

d. Una respuesta a las necesidades sociales y económicas del mercado. Salidas como el comercio exterior con idiomas deberían reflejarse específicamente en los itinerarios universitarios.

3. Utilidad de los foros. Los foros en Internet se posicionan como principal fuente de orientación actual. Además, parecen fomentar un espíritu de comunidad que a veces se echa de menos en las facultades. Los foros son herramientas que bien podrían utilizar las propias instituciones universitarias, con poco esfuerzo humano y económico de implantación, ofertando así un nuevo sistema de información adicional inmediato y eficiente.

Todas estas consideraciones, encaminadas a adaptar la formación al sujeto de la formación, redundarían en una mejora de la calidad universitaria, un aumento de la satisfacción del usuario y un enfoque muy mejorado de la proyección laboral y social de nuestra carrera.

\section{REFERENCIAS BIBLIOGRÁFICAS}

ARRÉs LÓPEZ, E. (2005) El traductor autónomo en España: requisitos, introducción en el mercado laboral y captación de clientes. Proyecto de Fin de Carrera: Universidad de Granada.

BIGGS, J (2003) Teaching for Quality in Higher Education, 2nd edition, Buckingham 
Calvo Encinas, E. Tesis doctoral (en fase de finalización). Análisis curricular de los estudios de Traducción e Interpretación desde la perspectiva del estudiantado. Universidad de Granada. Dirección a cargo de Dorothy Kelly.

----.(2006) "Orientación profesional para futuros licenciados de TeI: estrategias centradas en las necesidades del estudiantado". En Estudios de Traducción: Problemas y Perspectivas. Bravo Utrera, $S$ y García López, R. (Eds.) Universidad de Las Palmas de Gran Canaria: Servicio de Publicaciones.

GrotjAhn, R. (1987). "On the methodological basis of introspective methods”. En Færch, C. \& Kasper, G. (Eds.), Introspection in second language research. Clevedon, England: Multilingual Matters.

Kelly, DOROTHY (2003) "La investigación sobre formación de traductores: algunas reflexiones y propuestas". In Emilio Ortega Arjonilla (dir.) Panorama actual de la investigación en Traducción e Interpretación. Granada: Atrio. Vol I: 585-596

---- (2005) A Handbook for Translator Trainers. Manchester: St Jerome.

Mayoral Asensio, R. Ponencia invitada: "La formación de traductores: apuntes sobre su pasado, presente y futuro". En Seminario La formación de traductores en el contexto universitario: critica de la situación actual y algunas propuestas. Grupo de investigación AVANTI. 13 de febrero de 2004.

----. "Aspectos curriculares de la enseñanza de la traducción e interpretación en España”. En Aproximaciones a la traducción. Instituto Cervantes Virtual. Disponible en http://www.cvc.cervantes.es/obref/aproximaciones/mayoral.htm (30.11.06) 